03

\title{
Эллипсометрия металлических пленок в условиях аномального скин-эффекта
}

\author{
(C) А.Б. Сотский ${ }^{1}$, Е.А. Чудаков ${ }^{1}$, Л.И. Сотская ${ }^{2}$ \\ ${ }^{1}$ Могилевский государственный университет имени А.А. Кулешова, \\ 212022 Могилев, Республика Беларусь \\ ${ }^{2}$ Белорусско-Российский университет, \\ 212000 Могилев, Республика Беларусь \\ e-mail: ab_sotsky@mail.ru
}

Поступила в редакцию 26.02.2021 г.

В окончательной редакции 28.02.2021 г.

Принята к публикации 19.03.2021 г.

Сформулированы интегральные уравнения Фредгольма второго рода, описывающие поля волн ТЕ- и ТМ-поляризации в металлических пленках с учетом аномального скин-эффекта. Уравнения допускают численное решение методом квадратур. Исследованы электрические поля в золотых и алюминиевых пленках, расположенных на кремниевой подложке, и угловые зависимости поляризационных углов отраженного от пленок света. Установлено, что для решения обратной задачи многоугловой эллипсометрии металлических пленок с использованием приближения нормального скин-эффекта характерна нестабильность восстановленного комплексного показателя преломления металла при изменении толщины металлической пленки.

Ключевые слова: металлическая пленка, аномальный скин-эффект, интегральные уравнения, многоугловая эллипсометрия.

DOI: $10.21883 /$ OS.2021.07.51080.1847-21

\section{Введение}

При эллипсометрических исследованиях металлических пленок металл, как правило, рассматривается как среда с комплексной диэлектрической проницаемостью $\varepsilon$. Однако такой подход нельзя считать бездефектным, поскольку при решении обратных задач эллипсометрии он приводит к зависимости $\varepsilon$ от толщины пленки $[1,2]$. Данный подход основан на классической модели Друде-Зинера (модели нормального скин-эффекта), которая использует допущение о пространственно-локальной связи между плотностью тока свободных электронов $\mathbf{j}$ и напряженностью электрического поля $\mathbf{E}$. Но, как известно, такое допущение может быть корректным при условии, что средняя длина свободного пробега электрона много больше толщины скин-слоя [3]. Последнее условие может нарушаться в видимом и ультрафиолетовом диапазонах длин волн [3]. Кроме того, металлические пленки, используемые в микроэлектронике и оптике, часто имеют толщины, меньшие, чем длина свободного пробега электрона в металле. В этом случае допущение о локальной связи между векторами ј и $\mathbf{E}$ в пределах пленки также выглядит проблематичным.

Более корректное описание взаимодействия света с металлом, учитывающее пространственную нелокальность связи векторов $\mathbf{j}$ и $\mathbf{E}$, возможно в рамках теории аномального скин-эффекта, основанной на совместном рассмотрении уравнений Максвелла и кинетического уравнения Больцмана [4] и сводящей дело к решению интегро-дифференциальных уравнений для напряженности электрического поля в области металла $[3,4]$.
Но в замкнутом виде такое решение найдено только для случая нормального падения плоской волны на поверхность полуограниченного металла [3,4]. В ситуации, представляющей интерес для эллипсометрии, когда металлическая пленка конечной толщины освещается наклонно падающими волнами ТЕ- и ТМ-поляризаций теория аномального скин-эффекта существенно усложняется. В случае ТЕ-волн приближенные выражения для электрического поля в пленке и коэффициента отражения плоской волны от пленки предложены в $[5,6]$. В [5] интегро-дифференциальное уравнение аномального скин-эффекта для пленки на подложке сведено к неоднородному интегральному уравнению Фредгольма второго рода, которое исследовано в первом борновском приближении. В [6] решение интегро-дифференциального уравнения ищется в виде ряда Фурье, что оправдано лишь при рассмотрении периодической последовательности металлических пленок. Более сложный случай ТМ-волн c позиций аномального скин-эффекта изучен гораздо слабее и применительно к проблемам эллипсометрии до сих пор не рассматривался.

В настоящей работе развит подход, предложенный в [5]. Исследование проведено для случая диффузного рассеяния электронов проводимости границами пленки. Теория аномального скин-эффекта при наклонном падении волн ТЕ-, либо ТМ-поляризации на металлическую пленку, находящуюся на диэлектрической подложке, сведена к решению неоднородных интегральных уравнений Фредгольма второго рода относительно компонент электрического поля в пленке. В этих уравнениях за нор- 
мальный скин-эффект отвечают неоднородные части, а за аномальный скин-эффект - интегральные члены. Получены выражения для коэффициентов отражения волн TE- и ТМ-поляризаций от структуры окисел-металлическая пленка-подложка. Для численного решения интегральных уравнений использован метод квадратур. Оценена его внутренняя сходимость. Исследованы электрические поля в золотых и алюминиевых пленках, расположенных на кремниевой подложке. Установлено, что аномальный скин-эффект сказывается главным образом на распределении по толщине металлической пленки нормальной границам пленки составляющей электрического поля. Рассчитаны зависимости от угла падения света на пленку функций поляризационных углов $\operatorname{tg} \psi$ и $\cos \Delta$ для золотых и алюминиевых пленок различной толщины в условиях аномального скин-эффекта. Эти зависимости использованы как „экспериментальные“ в вычислительных экспериментах по решению обратной задачи многоугловой эллипсометрии в рамках стандартной модели нормального скин-эффекта. Расчеты показали нестабильность результатов решения обратной задачи при изменении толщин металлических пленок.

\section{Волны ТЕ-поляризации \\ В металлической пленке}

Пусть металлическая пленка толщины $d_{m}$ находится на подложке и отделена от воздуха диэлектрическим окисным слоем толщины $d_{o x}$ (рис. 1 ).

Монохроматическая плоская волна с зависимостью от времени $t$ и координаты $z$ вида $\exp \left(i \omega t-i k_{0} \beta z\right)$ (далее этот множитель в выражениях для компонент электромагнитного поля и плотности тока будет опускаться) падает на структуру из области $y>d_{o x}$ под углом $\gamma$. Здесь $\beta=\sqrt{\varepsilon_{a}} \sin \gamma, k_{0}=\omega / c-$ волновое число вакуума. Ось
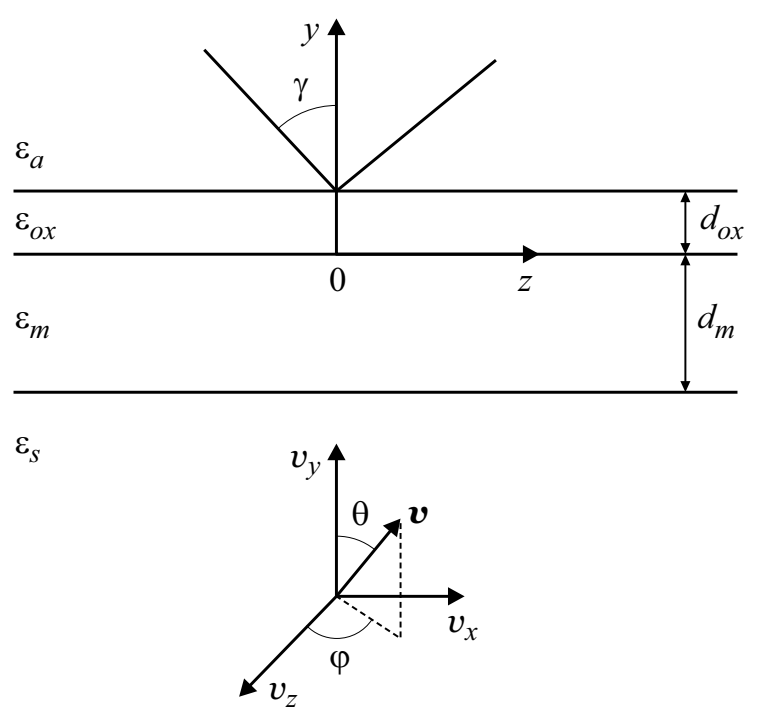

Рис. 1. Геометрия задачи и углы сферической системы координат в пространстве скорости электрона. однородности $0 x$. Относительные диэлектрические проницаемости подложки, металлической пленки, окисного слоя и воздуха равны $\varepsilon_{s}, \varepsilon_{m}, \varepsilon_{o x}, \varepsilon_{a}$. Здесь под $\varepsilon_{m}$ понимается диэлектрическая проницаемость ионного остова металла, учитывающая внутренний фотоэффект [3].

В соответствии с уравнениями Максвелла при возбуждении структуры волной ТЕ-поляризации единственная отличная от нуля компонента электрического поля $\mathbf{E}$ в металлической пленке подчиняется уравнению

$$
\frac{d^{2} E_{x}}{d y^{2}}+k_{0}^{2}\left[\varepsilon_{m}-\beta^{1}\right] E_{x}=J_{x},
$$

где

$$
\mathbf{J}=i \omega \mu_{0} \mathbf{j}+k_{0}^{2} \Delta \varepsilon \mathbf{E},
$$

$\Delta \varepsilon-$ некоторая константа, выбор которой пояснен ниже, $\mu_{0}$ - магнитная проницаемость вакуума (предполагается, что в рассматриваемом оптическом диапазоне частот магнитная проницаемость всех сред одинакова и равна $\left.\mu_{0}\right)$,

$$
\mathbf{j}=-e \int_{-\infty}^{\infty} d v_{x} \int_{-\infty}^{\infty} d v_{y} \int_{-\infty}^{\infty} d v_{z} \mathbf{v} f_{1}(y, \mathbf{v})
$$

- вектор плотности тока, $e$ - модуль заряда электрона, $f_{1}(y, \mathbf{v})$ - неравновесная функция плотности электронов в пространстве координат и скоростей.

Следуя [4], будем считать, что $f_{1}(y, \mathbf{v})$ подчиняется кинетическому уравнению Больцмана, записанному в приближении времени релаксации:

$$
\frac{\partial f_{1}}{\partial y}+k_{0} \alpha f_{1}=F
$$

где

$$
\begin{aligned}
& \alpha=\left[i \omega \tau\left(1-\beta v_{z} c^{-1}\right)+1\right]\left(\omega \tau v_{y} c^{-1}\right)^{-1}, \\
& F=\frac{e}{m v_{y}}\left(E_{x} \frac{\partial f_{0}}{\partial v_{x}}+E_{y} \frac{\partial f_{0}}{\partial v_{y}}+E_{z} \frac{\partial f_{0}}{\partial v_{z}}\right),
\end{aligned}
$$

$m$ - масса электрона, $\tau$ - среднее время свободного пробега электрона, $v_{x}, v_{y}, v_{z}$ - декартовы компоненты скорости электрона $\mathbf{v}$,

$$
f_{0}=\frac{2 m^{3}}{h^{3}} \frac{1}{\exp \left[\left(\varepsilon_{c}-\mu\right)(k T)^{-1}\right]+1}
$$

- равновесное распределение Ферми электронов по энергии $\varepsilon_{c}, h-$ постоянная Планка, $\mu=h^{2}(2 m)^{-1}\left(\sqrt[3]{3 n_{0}(8 \pi)^{-1}}\right)^{2}-$ предельная энергия Ферми, $n_{0}$ - концентрация свободных электронов, $k$ - постоянная Больцмана, $T-$ температура. Для рассматриваемых ТЕ-волн в (6) следует положить $E_{y}=E_{z}=0$.

Для однозначного решения уравнения (4) его надо дополнить граничными условиями для $f_{1}$ при $y=0$ и $y=-d$. Введем обозначения $f_{1}^{+}(y)=\left.f_{1}(y)\right|_{v_{y}>0}$, 
$f_{1}^{-}(y)=\left.f_{1}(y)\right|_{v_{y}<0}$. Функции $f_{1}^{+-}(y)$ должны удовлетворять уравнению (4) и граничным условиям [4]

$$
f_{1}^{+}(-d)=p_{1} f_{1}^{-}(-d), \quad f_{1}^{-}(0)=p_{2} f_{1}^{+}(0),
$$

где $p_{1}$ и $p_{2}-$ вероятности зеркального отражения электрона от границ пленки $y=-d_{o x}-d_{m}$ и $y=-d_{o x}$. Известно, что $p_{1}$ и $p_{2}$ могут заметно отличаться от нуля только в случае атомарно чистых поверхностей металлической пленки [6]. Такой чистоты поверхности сложно добиться при напылении пленок. Кроме того, в ряде работ, касающихся рассеяния электронов проводимости поверхностью металла, адекватной экспериментам считается модель диффузного рассеяния электронов поверхностью, в которой вероятность зеркального отражения электронов принимается равной нулю $[7,8]$. Руководствуясь данными соображениями, мы будем полагать $p_{1}=0, p_{2}=0$. В этом случае решения уравнения (4), удовлетворяющие граничным условиям (8), имеют вид

$$
\left.\begin{array}{l}
f_{1}^{+}(y)=\exp \left(-k_{0} \alpha y\right) \int_{-d}^{y} \exp \left(k_{0} \alpha y^{\prime}\right) F\left(y^{\prime}\right) d y^{\prime} \\
f_{1}^{-}(y)=\exp \left(-k_{0} \alpha y\right) \int_{0}^{y} \exp \left(k_{0} \alpha y^{\prime}\right) F\left(y^{\prime}\right) d y^{\prime}
\end{array}\right\} .
$$

Учитывая (3), (6), (9) и четность функции $f_{0}\left(v_{y}\right)$, находим

$$
\begin{aligned}
j_{x}(y)= & -\frac{e^{2}}{m} \int_{-\infty}^{\infty} d v_{x} \int_{0}^{\infty} d v_{y} \int_{-\infty}^{\infty} d v_{z} \frac{v_{x}}{v_{y}} \frac{\partial f_{0}}{\partial v_{x}} \\
& \times \int_{-d}^{0} \exp \left(-k_{0} \alpha\left|y-y^{\prime}\right|\right) E_{x}\left(y^{\prime}\right) d y^{\prime} .
\end{aligned}
$$

Переходя в (10) к сферическим координатам $v, \theta, \varphi$ в пространстве скорости $\mathbf{v}$ (рис. 1) и предполагая, что электронный газ вырожден, так что [4]

$$
\frac{\partial f_{0}}{\partial v_{x}}=-\frac{2 m^{3} \sin \theta \sin \varphi}{h^{3}} \delta\left(v-v_{0}\right),
$$

где $\delta\left(v-v_{0}\right) \quad-\quad$ дельта-функция Дирака, $v_{0}=h m^{-1} \sqrt[3]{3 n_{0}(8 \pi)^{-1}}-$ модуль скорости электрона на поверхности Ферми, можем записать формулу (10) в виде

$$
j_{x}(y)=\int_{-d}^{0} G_{x x}\left(y-y^{\prime}\right) E_{x}\left(y^{\prime}\right) d y^{\prime},
$$

где

$$
\begin{aligned}
G_{x x}\left(y-y^{\prime}\right)= & \frac{4 m e^{2} \mu^{2}}{h^{3}} \int_{0}^{2 \pi} d \varphi \sin ^{2} \varphi \int_{0}^{\pi / 2} d \theta \frac{\sin ^{3} \theta}{\cos \theta} \\
& \times \exp \left(-k_{0} \alpha\left|y-y^{\prime}\right|\right),
\end{aligned}
$$

$\alpha=\left[i \omega \tau\left(1-\beta v_{0} c^{-1} \sin \theta \cos \varphi\right)+1\right]\left(\omega \tau v_{0} c^{-1} \cos \theta\right)^{-1}$.
Решая (1) как неоднородное дифференциальное уравнение и учитывая (11), (12), приходим к интегральному уравнению

$$
\begin{aligned}
E_{x}(y)= & a \exp \left[i k_{0} k_{y}\left(y+d_{m}\right)\right]+b \exp \left[-i k_{0} k_{y}\left(y+d_{m}\right)\right] \\
& +\frac{k_{0} \Delta \varepsilon}{2 i k_{y}} \int_{-d}^{0} \exp \left(i k_{y} k_{0} \mid\left(y-y^{\prime} \mid\right) E_{x}\left(y^{\prime}\right) d y^{\prime}\right. \\
& +\frac{\omega \mu_{0}}{2 k_{y} k_{0}} \int_{-d}^{0} d y^{\prime} E_{x}\left(y^{\prime}\right) \int_{-d}^{0} \exp \left(i k_{y} k_{0} \mid\left(y-y^{\prime \prime} \mid\right)\right. \\
& \times G_{x x}\left(y^{\prime \prime}-y^{\prime}\right) d y^{\prime \prime},
\end{aligned}
$$

где $k_{y}=\sqrt{\varepsilon_{n}-\beta^{2}}, \varepsilon_{n}=\varepsilon_{m}+\Delta \varepsilon, a$ и $b-$ произвольные постоянные. После интегрирования по $y^{\prime \prime}$ уравнение (14) преобразуется следующим образом:

$$
\begin{aligned}
& E_{x}(y)=a \exp \left[i k_{0} k_{y}\left(y+d_{m}\right)\right]+b \exp \left[-i k_{0} k_{y}\left(y+d_{m}\right)\right] \\
& -i K \varepsilon_{n} \int_{0}^{2 \pi} d \varphi \int_{0}^{\pi / 2} d \theta \frac{\sin ^{3} \theta \sin ^{2} \varphi}{\left(\alpha^{2}+k_{y}^{2}\right) \cos \theta} \int_{-d}^{0} \exp \left(-\alpha k_{0} \mid\left(y-y^{\prime} \mid\right)\right. \\
& \times E_{x}\left(y^{\prime}\right) d y^{\prime}+A\left[\exp \left(i k_{y} k_{0} y\right) \int_{-d}^{y} \exp \left(-i k_{y} k_{0} y\right) E_{x}\left(y^{\prime}\right) d y^{\prime}\right. \\
& \left.-\exp \left(-i k_{y} k_{0} y\right) \int_{-d}^{y} \exp \left(i k_{y} k_{0} y\right) E_{x}\left(y^{\prime}\right) d y^{\prime}\right]
\end{aligned}
$$

где

$$
\begin{gathered}
K=-\frac{4 \omega \mu_{0} m e^{2} \mu}{k_{0}^{2} h^{3} \varepsilon_{n}} \\
A=-\frac{1}{k_{y}}\left[K \varepsilon_{n} \int_{0}^{2 \pi} d \varphi \int_{0}^{\pi / 2} \frac{\alpha \sin ^{3} \theta \sin ^{2} \varphi}{\left(\alpha^{2}+k_{y}^{2}\right) \cos \theta} d \theta+\frac{i k_{0} \Delta \varepsilon}{2}\right] .
\end{gathered}
$$

Положим в (15) $A=0$. Это условие конкретизирует константу $\Delta \varepsilon$ :

$$
\Delta \varepsilon=\frac{2 i K \varepsilon_{n}}{k_{0}} \int_{0}^{2 \pi} d \varphi \int_{0}^{\pi / 2} \frac{\alpha \sin ^{3} \theta \sin ^{2} \varphi}{\left(\alpha^{2}+k_{y}^{2}\right) \cos \theta} d \theta .
$$

Выражение (16) определяет вклад в комплексную диэлектрическую проницаемость металла электронов проводимости. Действительно, если, учитывая неравенство $v_{0} c^{-1} \ll 1$, пренебречь в (16) $k_{y}^{2}$ по сравнению с $\alpha^{2}$, а в формуле (5) пренебречь членом, содержащим $\beta$, то после элементарного интегрирования выражение (16) приобретает вид

$$
\Delta \varepsilon=-i \omega \mu_{0} \sigma\left[k_{0}^{2}(1+i \omega \tau)\right]^{-1},
$$


где

$$
\sigma=\frac{16 m e^{2} \pi \mu v_{0} \tau}{3 h^{3}}
$$

- статическая проводимость металла [10]. Формула (17), описывающая вклад свободных электронов в комплексную диэлектрическую проницаемость металла, может быть получена и в рамках классической теории Друде-Зинера [3]. Однако поскольку при низких частотах величина $k_{y}^{2}$ в (16) может быть сопоставимой с $\alpha^{2}$, для вычисления $\Delta \varepsilon$ целесообразно использовать точную формулу (16). Она допускает преобразование к виду

$$
\begin{aligned}
\Delta \varepsilon= & 2 \pi i K \varepsilon_{n} v_{0} \tau(1+i \omega \tau)^{-1} A^{2} \\
& \times\left[\left(1+A^{2}\right) A^{-1} \operatorname{arctg}\left(A^{-1}\right)-1\right]\left[1+O\left(\beta v_{0} c^{-1}\right)^{2}\right],
\end{aligned}
$$

где $A=(1+i \omega \tau)\left(k_{0} \tau v_{0} \sqrt{\varepsilon_{n}}\right)^{-1} \quad$ и $\quad$ выбрана $\quad$ ветвь $\left|\operatorname{Re}\left[\operatorname{arctg}\left(A^{-1}\right)\right] \leq 0.5 \pi\right|$. Из (18) очевидно, что зависимость $\Delta \varepsilon$ от $\beta$, или от угла падения излучения на металлическую пленку, не существенна.

Константы $a$ и $b$ в (15) находим, потребовав непрерывности тангенциальных составляющих электромагнитного поля на границе раздела металлическая пленка-подложка:

$$
\begin{gathered}
\left.E_{x}\right|_{y=-d_{m}-0}=\left.E_{x}\right|_{y=-d_{m}+0}, \\
d E_{x} /\left.d y\right|_{y=-d_{m}-0}=d E_{x} /\left.d y\right|_{y=-d_{m}+0} .
\end{gathered}
$$

В согласии с условием излучения Зоммерфельда в подложке

$$
d E_{x} /\left.d y\right|_{y=-d_{m}-0}=\left.i k_{0} \sigma_{s} E_{x}\right|_{y=-d_{m}-0}=i k_{0} \sigma_{s} E_{x}\left(-d_{m}\right),
$$

где $\sigma_{s}=\sqrt{\varepsilon_{s}-\beta^{2}}$. Определив правые части уравнений (19) из (15) и воспользовавшись (20), получаем итоговое интегральное уравнение относительно нормированной компоненты электрического поля $\bar{E}_{x}(y)=E_{x}(y) / E_{x}\left(-d_{m}\right)$ в металлической пленке:

$$
\begin{aligned}
& \bar{E}_{x}(y)=\bar{E}_{x}^{(n)}(y)+\Phi_{s}\left(y, k_{y}\right)+\Phi_{s}\left(y,-k_{y}\right) \\
& -i K \varepsilon_{n} \int_{0}^{2 \pi} d \varphi \int_{0}^{\pi / 2} d \theta \frac{\sin ^{3} \theta \sin ^{2} \varphi}{\left(\alpha^{2}+k_{y}^{2}\right) \cos \theta} \\
& \times \int_{-d}^{0} \exp \left(-\alpha k_{0}\left|y-y^{\prime}\right|\right) \bar{E}_{x}\left(y^{\prime}\right) d y^{\prime},
\end{aligned}
$$

где

$$
\bar{E}_{x}^{(n)}(y)=\cos \left[k_{0} k_{y}\left(y+d_{m}\right)\right]+i \sigma_{s} k_{y}^{-1} \sin \left[k_{0} k_{y}\left(y+d_{m}\right)\right],
$$

$$
\begin{aligned}
& \Phi_{s}\left(y, k_{y}\right)=\exp \left[k_{0} k_{y}\left(y+d_{m}\right)\right] \frac{K \varepsilon_{n}}{2 k_{y}} \int_{0}^{2 \pi} d \varphi \int_{0}^{\pi / 2} d \theta \\
& \times \frac{\sin ^{3} \theta \sin ^{2} \varphi}{\left(\alpha-i k_{y}\right) \cos \theta} \int_{-d_{m}}^{0} \exp \left(-\alpha k_{0}\left(y^{\prime}+d_{m} \mid\right) \bar{E}_{x}\left(y^{\prime}\right) d y^{\prime},\right.
\end{aligned}
$$

При условии задания величин $\bar{E}_{x}(0)$ и $\left(d \bar{E}_{x} / d y\right)_{y=0}$ коэффициент отражения ТЕ-волны от структуры, изображенной на рис. 1 , может быть рассчитан на основании рекуррентных соотношений [9]. Он равен

$$
r_{s}=\left(S S_{1}^{(1)}+S_{2}^{(2)}\right)\left(S S_{1}^{(2)}+S_{2}^{(1)}\right)^{-1},
$$

где

$$
\begin{gathered}
S=\left(d \bar{E}_{x} / d y\right)_{y=0}\left[k \bar{E}_{x}(0)\right]^{-1}, \\
S_{1}^{(1)}=i k_{y a} \sigma_{o x}^{-1} \sin \left(\sigma_{0 x} k_{0} d_{o x}\right)+(-1)^{l} \cos \left(\sigma_{0 x} k_{0} d_{o x}\right), \\
S_{2}^{(1)}=i k_{y a} \cos \left(\sigma_{o x} k_{0} d_{o x}\right)+(-1)^{l} \sin \left(\sigma_{o x} k_{0} d_{o x}\right),
\end{gathered}
$$

$k_{y a}=\sqrt{\varepsilon_{a}-\beta^{2}}, \sigma_{o x}=\sqrt{\varepsilon_{o x}-\beta^{2}}$. Величины $\bar{E}_{x}(0)$ и $\left(d \bar{E}_{x} / d y\right)_{y=0}$ вычисляются после решения уравнения (21). В частности, согласно (21),

$$
\begin{aligned}
& \frac{\left(d \bar{E}_{x} / d y\right)_{y=0}}{k_{0}}=i \sigma_{s} \cos \left(k_{y} k_{0} d_{m}\right)-k_{y} \sin \left(k_{y} k_{0} d_{m}\right) \\
& +k_{y}\left[\Phi_{s}\left(0, k_{y}\right)-\Phi_{s}\left(0,-k_{y}\right)\right]+i K \varepsilon_{n} \int_{0}^{2 \pi} d \varphi \int_{0}^{\pi / 2} d \theta \\
& \times \frac{\alpha \sin ^{3} \theta \sin ^{2} \varphi}{\left(\alpha^{2}+k_{y}^{2}\right) \cos \theta} \int_{-d_{m}}^{0} \exp \left(\alpha k_{0} y^{\prime}\right) \bar{E}_{x}\left(y^{\prime}\right) d y^{\prime} .
\end{aligned}
$$

Неоднородная часть уравнения Фредгольма $\bar{E}_{x}^{(n)}(y)$ описывает стоячую волну в металлической пленке, которая является решением задачи о нормальном скин-эффекте, имеющем место в пределе при $\omega \tau \rightarrow 0$ [3]. В этой задаче металлическая пленка рассматривается как среда с комплексной диэлектрической проницаемостью $\varepsilon_{n}=\varepsilon_{m}+\Delta \varepsilon$. Интегральные слагаемые в правой части (21) отвечают за аномальный скин-эффект, поскольку при $\omega \tau \rightarrow 0$ их ядра имеют порядок $O(\omega \tau)$ либо $O\left[(\omega \tau)^{2}\right]$ равномерно относительно $y$. Согласно (24) и (25), вклад аномального скин-эффекта в коэффициент отражения $r_{s}$ имеет порядок $O(\omega \tau)$.

Для численного решения уравнения (21) мы использовали метод квадратур [11]. В нем интервал $-d_{m} \leq y \leq 0$ разбивается на $N$ отрезков равной длины $\Delta y=d_{m} / N$. Координата левой границы $j$-го отрезка $(j=\overline{1, N})$ равна $y_{j}=-d_{m}+(j-1) \Delta y$. На данном отрезке для искомой функции $E(y)$ (в случае уравнения (21) $E_{y}=\bar{E}_{x}(y)$ ) используется линейная интерполяция

$$
E(y)=E_{j}+\left(E_{j+1}-E_{j}\right)\left(y-y_{j}\right) / \Delta y,
$$


где $E_{j}=E\left(y_{j}\right)$. Тогда внутренние интегралы в (22), (24) допускают аналитический расчет. В частности,

$$
\begin{aligned}
& \int_{-d_{m}}^{y_{i}} \exp \left[-\alpha k_{0}\left(y_{i}-y^{\prime}\right)\right] E\left(y^{\prime}\right) d y^{\prime}=V_{1}(i-2) E_{1} \\
& +V_{2} E_{i}+\sum_{j=2}^{i-1} V_{3}(i-j-1) E_{j}, \\
& \int_{y_{i}}^{0} \exp \left[-\alpha k_{0}\left(y^{\prime}-y_{i}\right)\right] E\left(y^{\prime}\right) d y^{\prime}=V_{1}(N-i) E_{n+1} \\
& +V_{2} E_{i}+\sum_{j=i+1}^{N} V_{3}(j-i-1) E_{j},
\end{aligned}
$$

где

$$
\begin{gathered}
V_{1}(I)=\exp \left(-k_{0} \alpha I \Delta y\right)\left[1-\exp \left(-k_{0} \alpha \Delta y\right)\right] /\left(k_{0}^{2} \alpha^{2} \Delta y\right), \\
V_{2}=\left[k_{0} \alpha \Delta y+\exp \left(-k_{0} \alpha \Delta y\right)-1\right] /\left(k_{0}^{2} \alpha^{2} \Delta y\right), \\
V_{3}(I)=\exp \left(-k_{0} \alpha I \Delta y\right)\left[1-\exp \left(-k_{0} \alpha \Delta y\right)\right]^{2} /\left(k_{0}^{2} \alpha^{2} \Delta y\right) .
\end{gathered}
$$

Придавая $\quad y \quad$ в (21) поочередно значения $y_{j}$ $(j=1, \ldots, N+1)$ и учитывая (27), (28), приходим к неоднородной алгебраической системе относительно значений искомой функции в узлах интерполяции:

$$
\sum_{l=1}^{N+1} M_{k l}^{(s)} E_{l}=E_{x}^{(n)}\left(y_{k}\right) \quad(k=1, \ldots, N+1) .
$$

Матричные элементы $M_{k l}^{(s)}$ представляются равномерно сходящимися относительно $\beta$ двойными интегралами по угловым переменным. Их явный вид нетрудно записать, подставив (27) и (28) в (21).

\section{Волны ТМ-поляризации В металлической пленке}

При возбуждении структуры, изображенной на рис. 1, волной ТМ-поляризации, во всем пространстве отличны от нуля компоненты электрического поля $E_{z}$ и $E_{y}$, а $E_{x} \equiv 0$. Тогда, в соответствии с уравнениями Максвелла в металлической пленке

$$
\begin{gathered}
\frac{d^{2} E_{z}}{d y^{2}}+k_{0}^{2} k_{y}^{2} E_{z}=\frac{1}{\varepsilon_{n}}\left(k_{y}^{2} J_{z}-\frac{i \beta}{k_{0}} \frac{d J_{y}}{d y}\right), \\
E_{y}=\frac{1}{k_{y}^{2}}\left(\frac{J_{y}}{k_{0}^{2}}-\frac{i \beta}{k_{0}} \frac{d E_{z}}{d y}\right),
\end{gathered}
$$

а на границах пленки $y=-d_{m}$ и $y=0$ должны быть непрерывны $E_{z}$ и величина $k_{0}^{-1} d E_{z} / d y+i \beta E_{y}$, пропорциональная компоненте магнитного поля $H_{x}$.
Повторение выкладок, аналогичных рассмотренным при анализе уравнения (1), позволяет свести интегро-дифференциальное уравнение (30) к интегральному уравнению

$$
\begin{aligned}
& \bar{E}_{z}(y)=\bar{E}_{z}^{(n)}(y)+\Phi_{p}\left(y, k_{y}\right)+\Phi_{p}\left(y,-k_{y}\right) \\
& -K \int_{0}^{2 \pi} d \varphi \int_{0}^{\pi / 2} d \theta \frac{\sin \theta\left(i k_{y}^{2} \sin \theta \cos \theta-\alpha \beta \cos \theta\right)}{\left(\alpha^{2}+k_{y}^{2}\right) \cos \theta} \\
& \times \int_{-d_{m}}^{0} \exp \left(-\alpha k_{0}\left|y-y^{\prime}\right|\right)\left[\bar{E}_{z}\left(y^{\prime}\right) \sin \theta \cos \varphi\right. \\
& \left.+\operatorname{sign}\left(y-y^{\prime}\right) \bar{E}_{y}\left(y^{\prime}\right) \cos \theta\right] d y^{\prime}
\end{aligned}
$$

где

$$
\begin{aligned}
& \bar{E}_{z}^{(n)}(y)=\cos \left[k_{0} k_{y}\left(y+d_{m}\right)\right]+\frac{i k_{y} \varepsilon_{s}}{\sigma_{s} \varepsilon_{n}} \sin \left[k_{0} k_{y}\left(y+d_{m}\right)\right], \\
& \Phi_{p}\left(y, k_{y}\right)=\exp \left[k_{0} k_{y}\left(y+d_{m}\right)\right] \\
& \times \frac{K}{2} \int_{0}^{2 \pi} d \varphi \int_{0}^{\pi / 2} d \theta \frac{\sin \theta\left(k_{y} \sin \theta \cos \varphi-\beta \cos \theta\right)}{\left(\alpha-i k_{y}\right) \cos \theta} \\
& \times \int_{-d_{m}}^{0} \exp \left[-\alpha k_{0}\left(y^{\prime}+d_{m}\right)\right]\left[\bar{E}_{z}\left(y^{\prime}\right) \sin \theta \cos \varphi\right. \\
& \left.-\bar{E}_{y}\left(y^{\prime}\right) \cos \theta\right] d y^{\prime}, \\
& \bar{E}_{z}(y)=E_{z}(y) / E_{z}\left(-d_{m}\right), \quad \bar{E}_{y}(y)=E_{y}(y) / E_{z}\left(-d_{m}\right) .
\end{aligned}
$$

Уравнение (32) содержит две неизвестных функции (35). Еще одно уравнение относительно этих функций получаем из (31), продифференцировав правую часть (32) по и воспользовавшись формулами (2), (3) и (6):

$$
\begin{aligned}
& \bar{E}_{y}(y)=\bar{E}_{y}^{(n)}(y)+\beta k_{y}^{-1}\left[\Phi_{p}\left(y, k_{y}\right)-\Phi_{p}\left(y,-k_{y}\right)\right] \\
& +\Delta \varepsilon \varepsilon_{n}^{-1} \bar{E}_{y}(y)-i K \int_{0}^{2 \pi} d \varphi \int_{0}^{\pi / 2} d \theta \\
& \times\left[1+\frac{\beta(\beta \cos \theta+i \alpha \sin \theta \cos \varphi)}{\left(\alpha^{2}+k_{y}^{2}\right) \cos \theta}\right] \sin \theta \\
& \times \int_{-d_{m}}^{0} \exp \left(-\alpha k_{0}\left|y-y^{\prime}\right|\right)\left[\bar{E}_{z}\left(y^{\prime}\right) \operatorname{sign}\left(y-y^{\prime}\right)\right. \\
& \left.\times \sin \theta \cos \varphi+\bar{E}_{y}\left(y^{\prime}\right) \cos \theta\right] d y^{\prime}
\end{aligned}
$$


где

$$
\begin{aligned}
\bar{E}_{y}^{(n)}(y)= & \frac{\beta}{k_{y}}\left\{i \sin \left[k_{0} k_{y}\left(y+d_{m}\right)\right]+\frac{k_{y} \varepsilon_{s}}{\sigma_{s} \varepsilon_{n}}\right. \\
& \left.\times \cos \left[k_{0} k_{y}\left(y+d_{m}\right)\right]\right\} .
\end{aligned}
$$

Выражения (32), (36) представляют собой замкнутую систему неоднородных интегральных уравнений Фредгольма второго рода, описывающих поле ТМ-поляризации в металлической пленке.

Заметим, что функции $\bar{E}_{z}^{(n)}(y)$ и $\bar{E}_{y}^{(n)}(y)$ вида (33) и (37) представляют собой компоненты электромагнитного поля, найденные в приближении нормального скин-эффекта. При рассмотрении уравнения (32) это достаточно очевидно, поскольку в пределе при $\omega \tau \rightarrow 0$ ядра интегралов в правой части (32) имеют порядок $O(\omega \tau)$ либо $O\left[(\omega \tau)^{2}\right]$ равномерно относительно $y$. Порядок $O(\omega \tau)$ имеют и функции $\Phi_{p}\left(y, \pm k_{y}\right)$ в (36). Но в случае уравнения (36) предел $\lim _{\omega \tau \rightarrow 0} \bar{E}_{y}(y)=\bar{E}_{y}^{(n)}(y)$ достигается, если только точка наблюдения $y$ находится внутри пленки. Действительно, если в (36) считать изменение функций $\bar{E}_{z}\left(y^{\prime}\right), \bar{E}_{y}\left(y^{\prime}\right)$ на интервале интегрирования $\left|y^{\prime}-y\right| \sim v_{0} \tau$ пренебрежимо малым [3], то в пределе при $\omega \tau \rightarrow 0$ или $\alpha \rightarrow \infty$ (см. (5))

$$
\int_{-d_{m}}^{0} \exp \left(-\alpha k_{0}\left|y-y^{\prime}\right|\right)\left[\bar{E}_{z}\left(y^{\prime}\right) \operatorname{sign}\left(y-y^{\prime}\right) d y^{\prime}=O\left[(\omega \tau)^{2}\right],\right.
$$$$
\int_{-d_{m}}^{0} \exp \left(-\alpha k_{0}\left|y-y^{\prime}\right|\right)\left[\bar{E}_{y}\left(y^{\prime}\right) d y^{\prime}=\frac{2 \bar{E}_{y}(y)}{\alpha k_{0}}+O\left[(\omega \tau)^{2}\right] .\right.
$$

При выполнении (38), (39) два последних слагаемых в правой части (36) сокращаются. Однако если $y \rightarrow-d_{m}$ либо $y \rightarrow 0$, то равенства (38), (39) нарушаются. В результате вблизи границ металлической пленки компонента электрического поля $E_{y}$ может существенно отклоняться от результата теории нормального скин-эффекта. Ниже это подтверждено численными расчетами.

Формула для коэффициента отражения плоской волны ТМ-поляризации от структуры, приведенной на рис. 1, получается аналогично (24) и имеет вид

$$
r_{p}=\left(P P_{1}^{(2)}+P_{2}^{(1)}\right)\left(P P_{1}^{(1)}+P_{2}^{(2)}\right)^{-1},
$$

где

$$
\begin{gathered}
P=-\left[k_{0}^{-1}\left(d \bar{E}_{z} / d y\right)_{y=0}+i \beta \bar{E}_{y}(0)\right] / \bar{E}_{z}(0), \\
P_{1}^{(l)}=\cos \left(\sigma_{o x} k_{0} d_{o x}\right)+(-1)^{l} \frac{\varepsilon_{a} \sigma_{o x}}{\varepsilon_{o x} i k_{y a}} \sin \left(\sigma_{o x} k_{0} d_{o x}\right), \\
P_{2}^{(l)}=\frac{\varepsilon_{o x}}{\sigma_{o x}} \sin \left(\sigma_{0 x} k_{0} d_{o x}\right)+(-1)^{l} \frac{\varepsilon_{a}}{i k_{y a}} \cos \left(\sigma_{0 x} k_{0} d_{o x}\right) .
\end{gathered}
$$

Коэффициент $P$ вычисляется после решения уравнений (32), (36). В частности, дифференцируя правую часть уравнения (32) и учитывая (36), находим

$$
\begin{aligned}
& k_{0}^{-1}\left(\bar{E}_{z} / d y\right)_{y=0}+i \beta \bar{E}_{y}(0)=i \varepsilon_{n} \beta^{-1} \bar{E}_{y}^{(n)}(0)+i \varepsilon_{n} k_{y}^{-1} \\
& \times\left[\Phi_{p}\left(0, k_{y}\right)-\Phi_{p}\left(0,-k_{y}\right)\right]+K \varepsilon_{n} \int_{0}^{2 \pi} d \varphi \int_{0}^{\pi / 2} d \theta \\
& \times \frac{i \alpha \sin ^{2} \theta \cos \varphi+\beta \cos \theta \sin \theta}{\left(\alpha^{2}+k_{y}^{2}\right) \cos \theta} \int_{-d_{m}}^{0} \exp \left(\alpha k_{0} y^{\prime}\right)\left[\bar{E}_{z}\left(y^{\prime}\right)\right. \\
& \left.\times \sin \theta \cos \varphi+\bar{E}_{y}\left(y^{\prime}\right) \cos \theta\right] d y^{\prime} .
\end{aligned}
$$

Как следует из (40) и (41), при $\omega \tau \rightarrow 0$ вклад аномального скин-эффекта в коэффициент отражения $r_{p}$ имеет порядок $O(\omega \tau)$.

Использование интерполяции (26) для функций $\bar{E}_{z}(y)$ и $\bar{E}_{y}(y)$ позволяет свести уравнения (32), (36) к неоднородной алгебраической системе

$$
\sum_{l=1}^{2(N+1)} M_{k l}^{(p)} X_{l}=B_{k} \quad(k=1, \ldots, 2(N+1)) .
$$

Здесь $X_{l}=\bar{E}_{z}\left(y_{l}\right), B_{l}=\bar{E}_{z}^{(n)}\left(y_{l}\right)$ при $l=1, \ldots, N+1$; $X_{l}=\bar{E}_{y}\left(y_{l-N-1}\right), B_{l}=\bar{E}_{y}^{(n)}\left(y_{l-N-1}\right)$, при $l=N+2, \ldots$, $\ldots 2(N+1)$. Матричные элементы $M_{k l}^{(p)}$ представляются равномерно сходящимися относительно $\beta$ двойными интегралами по угловым переменным. Их явный вид легко найти, подставив (27) и (28) в (32), (34), (36).

\section{Результаты расчетов}

На основании разработанной вычислительной схемы мы рассчитали поля волн ТЕ- и ТМ-поляризаций в металлических пленках и зависимости функций поляризационных углов $\operatorname{tg} \psi$ и $\cos \Delta$ от угла падения $\gamma$. Здесь

$$
\operatorname{tg} \psi=\left|r_{p} r_{s}\right|^{-1}, \quad \cos \Delta=\operatorname{Re}\left(r_{p} r_{s}^{-1}\right) / \operatorname{tg} \psi .
$$

Исследованы золотые и алюминиевые пленки на длине волны $\lambda=800 \mathrm{~nm}$, находящиеся на кремниевой подложке $\left(\varepsilon_{a}=1.0003^{2}, \varepsilon_{s}=(3.695-i 0.0066)^{2}[12]\right)$. Использованы константы металлов, приведенные в табл. 1, где $n_{m}-i k_{m}=\sqrt{\varepsilon_{n}}$. Как видно из табл. 1 , оптические

Таблица 1. Константы металлов, использованные при расчетах [13-15]

\begin{tabular}{c|c|c|c|c}
\hline Металл & $\tau, \mathrm{fs}$ & $n_{0} \cdot 10^{-28}, \mathrm{~m}^{-3}$ & $n_{m}$ & $k_{m}$ \\
\hline $\mathrm{Au}$ & 27.1 & 5.9 & 0.153 & 4.908 \\
$\mathrm{Al}$ & 7.2 & 18.07 & 2.767 & 8.354
\end{tabular}


Таблица 2. Внутренняя сходимость вычислительной схемы

\begin{tabular}{r|c|c|c|c|c|c}
\hline $\mathrm{N}$ & $\operatorname{tg} \psi$ & $\operatorname{tg} \psi_{0}$ & $\operatorname{tg} \psi_{n}$ & $\cos \Delta$ & $\cos \Delta_{0}$ & $\cos \Delta_{n}$ \\
\hline 50 & 0.957905 & - & & -0.578713 & - & \\
100 & 0.958067 & 0.958121 & & -0.578778 & -0.578800 & \\
150 & 0.958109 & 0.958142 & 0.963019 & -0.578792 & -0.578803 & -0.578253 \\
200 & 0.958125 & 0.958148 & & -0.578797 & -0.578804 & \\
250 & 0.958133 & 0.958147 & & -0.578800 & -0.578804 & \\
300 & 0.958137 & 0.958147 & & -0.578800 & -0.578804 &
\end{tabular}
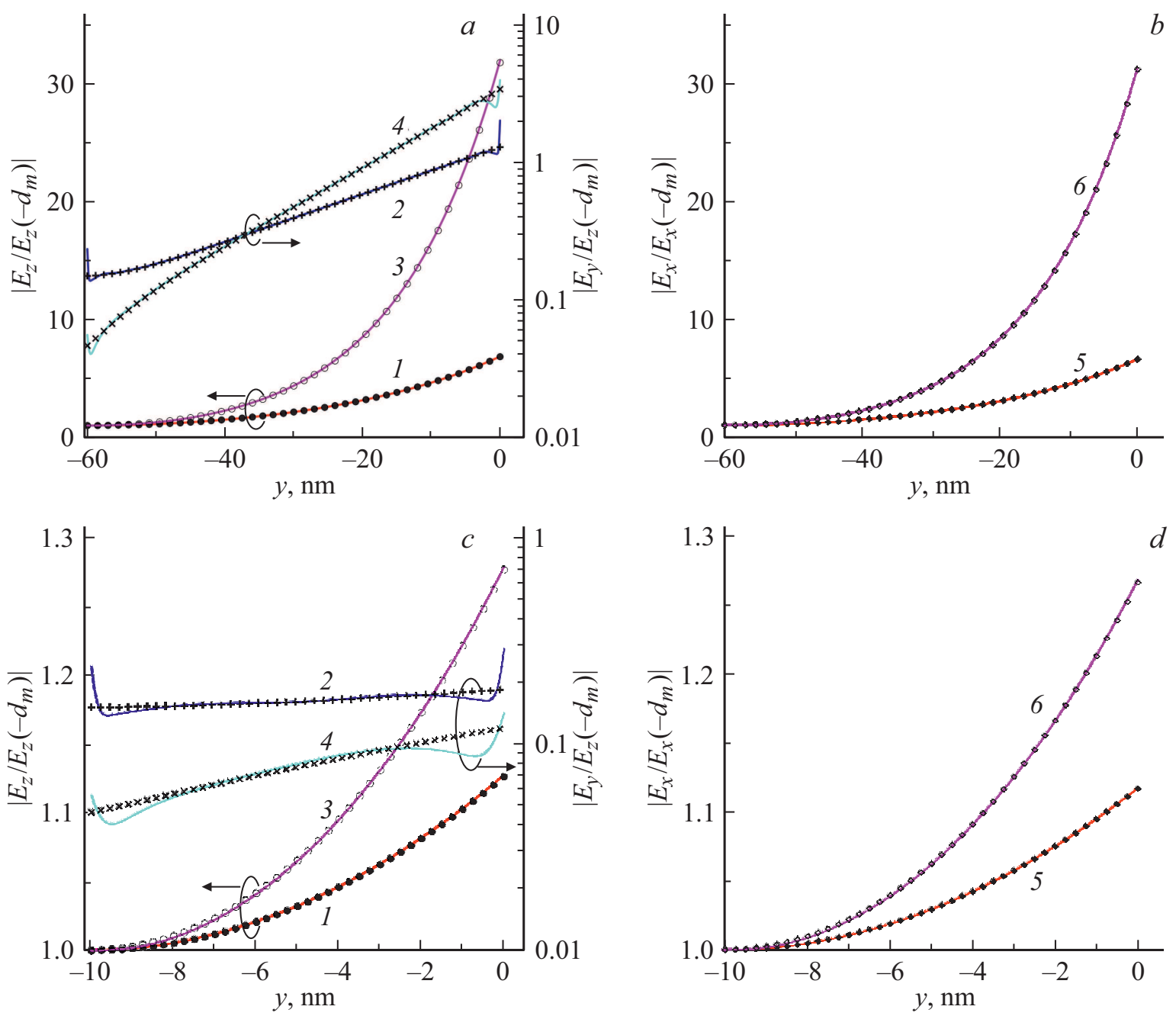

Рис. 2. Распределения компонент электрического поля в золотой $(1,2,5)$ и алюминиевой $(3,4,6)$ пленках толщиной $60(a, b)$ и $10 \mathrm{~nm}(c, d)$ для волн ТМ- $(a, c)$ и ТЕ-поляризаций $(b, d)$. Сплошные кривые - модель аномального скин-эффекта, дискретные значки - модель нормального скин-эффекта.

константы золота и алюминия существенно различаются. Выбор пленок из этих материалов объясняется, с одной стороны, их широким использованием в оптике и микроэлектронике, а с другой, возможностью оценить влияние аномального скин-эффекта на оптические поля в исследуемой структуре (рис. 1) при различных условиях, в том числе и при наличии окисного слоя на поверхности алюминиевой пленки.
Таблица 2 иллюстрирует внутреннюю сходимость разработанной вычислительной схемы. Она относится к золотой пленке с $d_{m}=60 \mathrm{~nm}$ и соответствует $d_{o x}=0$, $\gamma=70^{\circ}$.

Известно, что погрешность метода квадратур при решении интегральных уравнений имеет такой же порядок, как и погрешность принятой квадратурной формулы при вычислении интегралов в интегральных уравнениях [11]. 

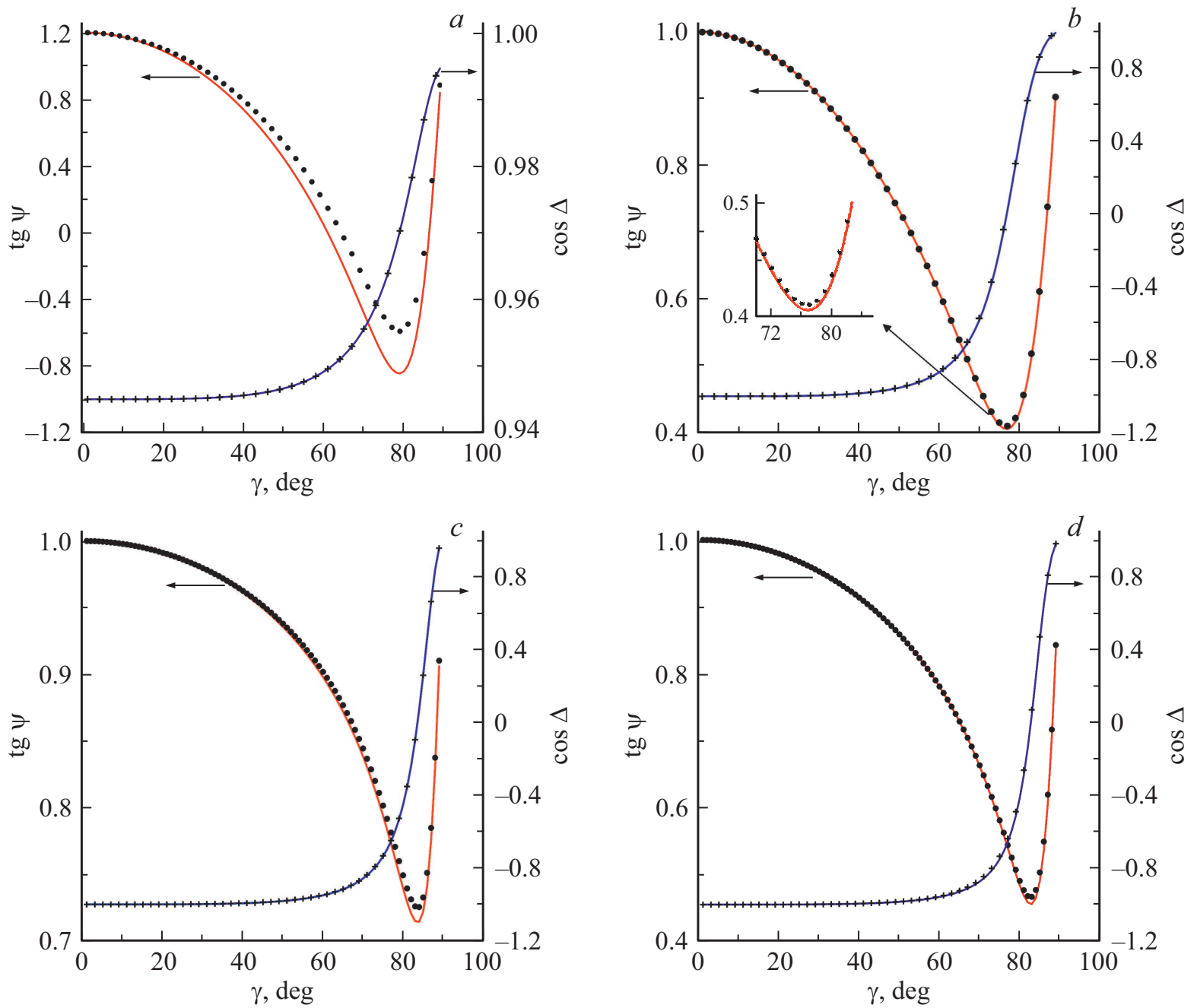

Рис. 3. Угловые зависимости функций поляризационных углов для золотых $(a, b)$ и алюминиевых $(c, d)$ пленок на кремниевой подложке при $d_{o x}=0 \mathrm{~nm}: a, c-d_{m}=60 \mathrm{~nm} ; b, d-d_{m}=10 \mathrm{~nm}$. Сплошные кривые - теория аномального скин-эффекта, дискретные значки - приближение нормального скин-эффекта.

Поэтому в случае линейной интерполяции (26)

$$
f(N)=f_{0}+C / N^{2}+O\left(1 / N^{3}\right),
$$

где $C$ - некоторая константа, $f_{0}=\lim _{N \rightarrow \infty} f(N)-$ точное решение задачи, а под $f$ понимается $\operatorname{tg} \psi$ либо $\cos \Delta$. Выражение (43) может быть использовано для ускорения сходимости вычислительной схемы. Действительно, если рассчитать значения $f\left(N_{1}\right), f\left(N_{2}\right)$ и пренебречь в (43) величинами порядка $O\left(1 / N_{1,2}^{3}\right)$, то из (43) найдем

$$
f_{0}=f\left(N_{1}\right)+N_{2}^{2}\left[f\left(N_{2}\right)-f\left(N_{1}\right)\right]\left(N_{2}^{2}-N_{1}^{2}\right)^{-1} .
$$

В таблице 2 под $\operatorname{tg} \psi$ и $\cos \Delta_{0}$ понимаются величины, pacсчитанные на основании (44) при $N_{2}=N, N_{1}=N-50$, а через $\operatorname{tg} \psi_{n}$ и $\cos \Delta_{n}$ обозначены $\operatorname{tg} \psi$ и $\cos \Delta$, найденные в приближении нормального скин-эффекта, т.е. при использовании в $(24)$ и $(40)$

$$
\frac{\left(d \bar{E}_{x} / d y\right)_{y=0}}{k_{0}}=i \sigma_{s} \cos \left(k_{y} k_{0} d_{m}\right)-k_{y} \sin \left(k_{y} k_{0} d_{m}\right),
$$

$$
\begin{gathered}
\bar{E}_{x}(0)=\bar{E}_{x}^{(n)}(0), \\
k_{0}^{-1}\left(d \bar{E}_{z} / d y\right)_{y=0}+i \beta \bar{E}_{y}(0)=i k_{y}^{2} \beta^{-1} \bar{E}_{y}^{(n)}(0), \\
\bar{E}_{z}(0)=\bar{E}_{z}^{(n)}(0) .
\end{gathered}
$$

Данные табл. 2 подтверждают эффективность алгоритма (44). Еще в табл. 2 можно отметить заметное расхождение значений $\operatorname{tg} \psi$, рассчитанных в приближениях аномального и нормального скин-эффектов.

На рис. 2 представлены распределения компонент электрического поля в золотых и алюминиевых пленках, возбуждаемых волнами ТЕ- и ТМ-поляризаций. Они соответствуют $N \geq 50, d_{o x}=0, \gamma=70^{\circ}$.

На рис. 2 распределения $\left|\bar{E}_{x}(y)\right|,\left|\bar{E}_{z}(y)\right|$, рассчитанные в рамках моделей нормального и аномального скин-эффектов, визуально неотличимы. Однако имеет место ожидаемое, согласно предыдущему разделу, заметное расхождение аналогичных распределений $\left|\bar{E}_{y}(y)\right|$ в окрестности границ металлических пленок 


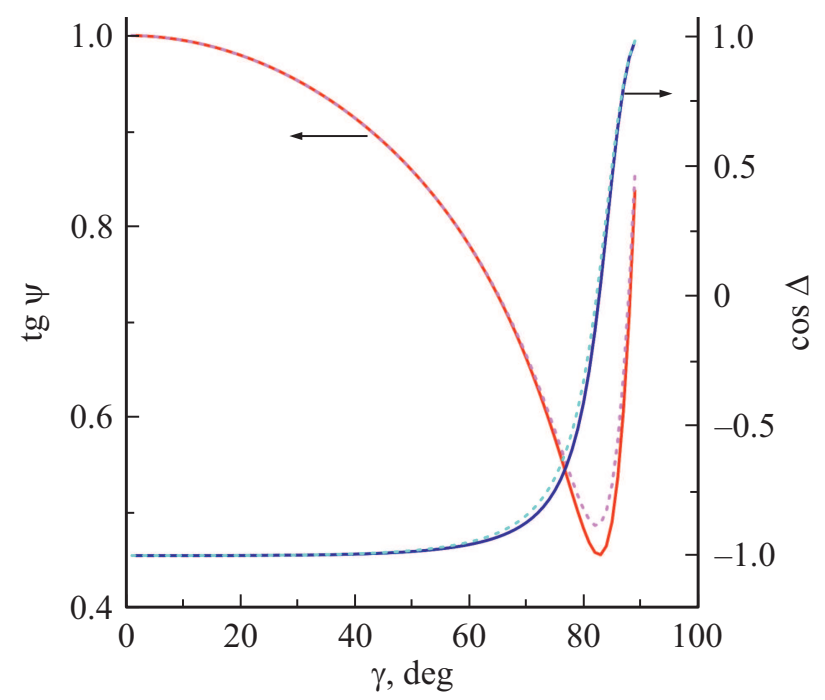

Рис. 4. Угловые зависимости функций поляризационных углов для алюминиевой пленки толщиной $d_{m}=10 \mathrm{~nm}$ на кремниевой подложке при наличии (штриховые кривые) и отсутствии (сплошные кривые) на границе раздела пленка-воздух окисного слоя с показателем преломления $n_{o x}=1.7601$ и толщиной $d_{o x}=2 \mathrm{~nm}$.

(на рис. 2, a, c распределению $\left|\bar{E}_{x}(y)\right|$ в случае золотой пленки отвечают значки „+“", а в случае алюминиевой пленки - „х“). Это расхождение возрастает при уменьшении толщины пленки. Аналогичная тенденция в случае ТЕ-волн ранее отмечалась в [5].

Влияние аномального скин-эффекта на функции поляризационных углов иллюстрирует рис. 3 .

На рис. 3 расхождение результатов приближений нормального и аномального скин-эффектов наблюдается главным образом для функции $\operatorname{tg} \psi(\gamma)$.

Рисунок 4 иллюстрирует трансформацию распределений $\operatorname{tg} \psi$ и $\cos \Delta(\gamma)$, рассчитанных в рамках модели аномального скин-эффекта, при образовании окисного слоя на поверхности алюминиевой пленки.

Таблица 3 позволяет судить о погрешностях решения обратной задачи многоугловой эллипсометрии в случае использования стандартной модели нормального скин-эффекта. В таблице $d_{m}$ и $d_{o x}$ - точные толщины металлической пленки и окисного слоя; $d_{m}^{(r)}, n_{m}^{(r)}$, $k_{m}^{(r)}, d_{o x}^{(r)}, n_{o x}^{(r)}-$ соответственно толщина, показатель преломления, показатель поглощения металлической пленки, толщина и показатель преломления окисного слоя, определенные путем минимизации градиентным методом целевой функции

$$
I=\sum_{i=0}^{l}\left[\operatorname{tg} \psi\left(\gamma_{i}\right)-\operatorname{tg} \psi_{n}\left(\gamma_{i}\right)\right]^{2}+\left[\cos \Delta\left(\gamma_{i}\right)-\cos \Delta_{n}\left(\gamma_{i}\right)\right]^{2},
$$

где $\gamma_{i}=i \Delta \gamma, \Delta \gamma=1^{\circ}, l=89, \operatorname{tg} \psi\left(\gamma_{i}\right), \cos \Delta\left(\gamma_{i}\right)-$ функции поляризационных углов, рассчитанные в рамках модели аномального скин-эффекта при значениях констант, указанных в табл. 1. Графики данных функций приведены
Таблица 3. Решение обратной задачи многоугловой эллипсометрии в приближении нормального скин-эффекта

\begin{tabular}{c|c|c|c|c|c|c|c}
\hline Пленка & $\begin{array}{c}d_{m}, \\
\mathrm{~nm}\end{array}$ & $\begin{array}{c}d_{o x}, \\
\mathrm{~nm}\end{array}$ & $\begin{array}{c}d_{m}^{(r)}, \\
\mathrm{nm}\end{array}$ & $n_{m}^{(r)}$ & $k_{m}^{(r)}$ & $\begin{array}{c}d_{o x}^{(r)}, \\
\mathrm{nm}\end{array}$ & $n_{o x}^{(r)}$ \\
\hline \multirow{2}{*}{$\mathrm{Au}$} & 60 & 0 & 60.04 & 0.190 & 4.906 & 0 & - \\
\cline { 2 - 8 } & 10 & 0 & 10.00 & 0.235 & 4.896 & 0 & - \\
\hline \multirow{2}{*}{$\mathrm{Al}$} & 60 & 0 & 54.03 & 2.897 & 8.336 & 0 & - \\
\cline { 2 - 9 } & 10 & 0 & 10.07 & 2.972 & 8.347 & 0 & - \\
\hline \multirow{2}{*}{$\mathrm{Al}$} & 60 & 2 & 59.61 & 2.881 & 8.317 & 1.92 & 1.7635 \\
\cline { 2 - 8 } & 10 & 2 & 10.03 & 2.925 & 8.355 & 1.95 & 1.6860
\end{tabular}

на рис. 3 , 4. В (45) функциями параметров $d_{m}^{(r)}, n_{r}, k_{r}$, $d_{o x}^{(r)}, n_{o x}^{(r)}$ являются $\operatorname{tg} \psi_{n}\left(\gamma_{i}\right)$ и $\cos \Delta_{n}\left(\gamma_{i}\right)$.

Как видно из табл. 3 , основной дефект использования модели нормального скин-эффекта при решении обратной задачи многоугловой эллипсометрии металлической пленки состоит в нестабильности значений $n_{m}^{(r)}$, относящихся к пленкам разной толщины. Кроме того, среди всех восстановленных параметров структур именно для $n_{m}^{(r)}$ характерно наиболее заметное отклонение от исходных констант пленок, приведенных в табл. 1.

\section{Заключение}

Сформулированы неоднородные интегральные уравнения Фредгольма второго рода, описывающие аномальный скин-эффект в металлической пленке для волн ТЕи ТМ-поляризации. Численное решение уравнений получено методом квадратур. Оценены погрешности стандартного приближения нормального скин-эффекта при расчете электрических полей в золотых и алюминиевых пленках и при решении обратной задачи многоугловой эллипсометрии для данных пленок.

Представляет интерес решение обратных оптических задач об определении параметров металлических пленок с использованием развитой теории аномального скин-эффекта. Стимулом к разработке соответствующих алгоритмов является нестабильность восстанавливаемой комплексной диэлектрической проницаемости при изменении толщины металлических пленок, которая имеет место при решении обратных оптических задач с использованием модели нормального скин-эффекта. В случае многоугловой эллипсометрии этот дефект проиллюстрирован расчетами настоящей работы. Согласно экспериментальным данным [1,2,16], он наблюдается также при спектральной эллипсометрии и спектрофотометрии пленок из различных металлов. Применение для решения обратных оптических задач эллипсометрии и спектрофотометрии металлических пленок разработанной модели аномального скин-эффекта может открыть возможности раздельной оценки вкладов в комплексную 
диэлектрическую проницаемость металла $\varepsilon_{n}=\varepsilon_{m}+\Delta \varepsilon$ его ионного остова $\left(\varepsilon_{m}\right)$ и электронов проводимости $(\Delta \varepsilon$, формула (18)) и определить характеристики электронного газа $\mu, \tau, v_{0}$. В случае обработки экспериментальных данных, полученных методами спектральной эллипсометрии и спектрофотометрии соответствующие расчеты предполагают использование некоторой дисперсионной модели для функции $\varepsilon_{m}(\lambda)$ (например, модели Лоренца [1]). Однако численный расчет значений $r_{s}$ и $r_{p}$ при фиксированных $\gamma$ и $\lambda$ на персональном компьютере с использованием сформулированного выше алгоритма занимает около минуты, что затрудняет его использование для решения обратных оптических задач. Данные задачи требуют дополнительных исследований по оптимизации алгоритма.

\section{Финансирование работы}

Работа выполнена при финансовой поддержке Государственной программы научных исследований Республики Беларусь 1.15 „Фотоника и электроника для инновацийс.

\section{Конфликт интересов}

У авторов конфликта интересов нет.

\section{Список литературы}

[1] Lin J., Jiang H., Gu H., Chen X., Zhang C., Liao G., Liu S. // Physica Scripta. 2019. V. 94. P. 085802-1. doi 10.1088/1402-4896/ab1606

[2] Минкович В.П., Сотский А.Б., Шилов А.В., Сотская Л.И. // Журнал прикл. спектр. 2019. Т. 86. № 1. C. 128; Minkovich V.P., Sotsky A.B., Shilov A.V., Sotskaya L.I. // J. Applied Spectroscopy. 2019. V. 86. N 1. P. 112. doi 0021-9037/19/8601-0112

[3] Соколов А.В. Оптические свойства металлов. М: ГИФМЛ, 1961. $464 \mathrm{c}$

[4] Reuter G.E.H., Sondheimer E.H. // Proc. R. Soc. Lond. 1948. V. 195. P. 336. doi 10.1098/rspa.1948.0123

[5] Сотский А.Б., Парашков С.О., Василенко А.Н. // Проблемы физики, математики и техники. 2015. Т. 22. № 1. С. 21.

[6] Iakushev D., Lopez-Aguayo S. // JOSA B. 2017. V. 34. N 11. P. 2421. doi: 10.1364/JOSAB.34.002421

[7] Bennett H.E., Bennett J.M., Ashley E.J., Motyka R.J. // Phys. Rev. 1968. V. 165. N 3. P. 755-765. doi 10.1103/PhysRev.165.755

[8] Dingle R.B., Dingle R.B. // Physica. 1953. V. 19. Issues 1-12. P. 348. doi 10.1016/S0031-8914(53)80036-4

[9] Сотский А.Б. Теория оптических волноводных элементов. Могилев: УО „МГУ им. А.А. Кулешова“. 2011. 456 с.

[10] Румер Ю.Б., Рывкин М.Ш. Термодинамика, статистическая физика и кинетика. М.: Наука, 1972. 400 с.

[11] Крылов В.И., Бобков В.В., Монастырный П.И. Вычислительные методы. Т. 2. М.: Наука, 1977. 399 с.

[12] Palik E.D. Handbook of Optical Constants of Solids. Washington, D.C.: Naval Research Laboratory, 1985. P. 547. doi 10.1016/C2009-0-20920-2
[13] Johnson P.B., Christy R.W. // Phys. Rev. B. 1972. V. 6. N 12. P. 4370. doi 10.1103/physrevb.6.4370

[14] Rakić A.D. // Applied Optics. 1995. V. 34. N 22. P. 4755. doi 10.1364/AO.34.004755

[15] Echániz T., Pérez-Sáez R.B., Tello M.J. // J. Appl. Phys. 2014. V. 116. P. 093508. doi 10.1063/1.4894169

[16] Kangarlow H., Aghgonband M.M. // Opt. Spectrosc. 2013. V. 115. N 5. P. 753. doi 10.1134/S0030400X13110088 